\title{
ON THE PARAMETRIC TRANSVERSE VIBRATIONS OF CONTINUOU'S BEAM ON INTERMEDIATE ELASTIC SUPPORTS UNDER THE ACTION OF MOVING BODY
}

\author{
DO XUAN THO - NGUYEN VAN KHANG \\ Hanoi University of Technology
}

\begin{abstract}
SUMMARY. In this text the method of substructures [2] is applied for establishing the vibration equation of beam under the action of moving body. Later an algorithm is built to solve the vibration equation received. From this algorithm a computer program is set up with TURBO PASCAL 6.0 language
\end{abstract}

\section{INTRUDUCTION}

Parametric transverse vibration of continuous beam with intermediate elastic supports under the action of moving body has been mentioned in some works such as $[1,3,4]$. In this text the method of substructures [2] is applied for establishing the vibration equation of beam under the action of moving body. Later an algorithm is built to solve the vibration equation received. From this algorithm a computer program is set up with TURBO PASCAL 6.0 language.

\section{SETTTING UP THE VIBRATION EQUATION}

Consider a continuous beam with $n g$ intermediate elastic supports and span $\ell$. Suppose that its mass of length unit is $\rho F$ and bending rigidity $E J$ is constant on all of its length. Here $\rho$ is mass density, $F$ - cross sectional area, $E$ - elastic module, $J$ - moment of inertia. $C_{i}$ and $\ell_{i}$ are called $(i=1, \ldots, n g)$ respectively rigidity and coordinate of intermediate elastic supports $i$.

The body consists of mass $m$ attached to the spring system with rigidity $k$ and a damper system $d$ directly proportional with velocity. Body moves with velocity $v$ and bears the action of force $G \sin \Omega t$ caused by the disequilibrium mass which rotates with angle speed $\Omega$. Here $G$ is amplitude of force. Besides it is supposed that during all the moving time, body is not separated from the beam (fig. 2.1).

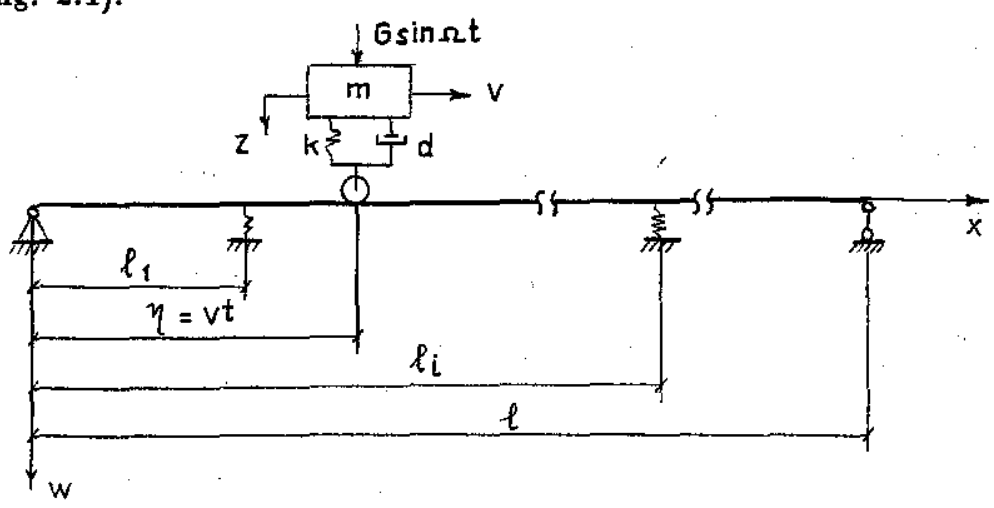

Fig. 2.1 


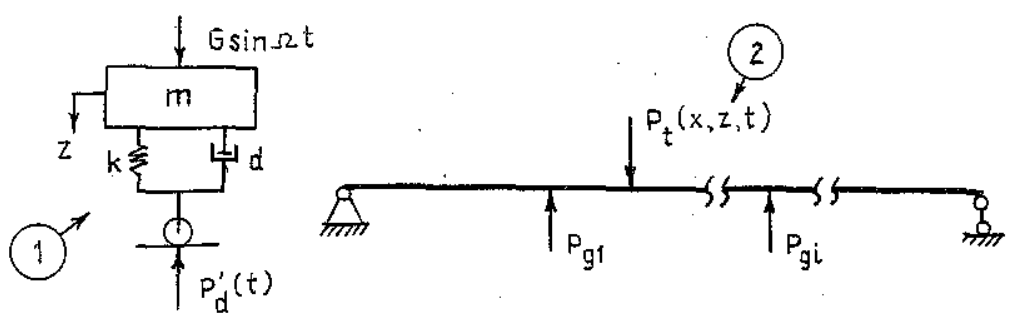

Fig. 2.2

Substructure 2 is considered a simple one bearing the following forces:

1. Pressure $P_{\tau}(x, z, t)$ of load on beam

$$
P_{\tau}(x, z, t)=[m g+G \sin \Omega t-(m \ddot{z}+d \dot{z}+k z)] \delta(x-v t)
$$

in which $g$ - gravity acceleration, $\delta(x)$ - Dirac delta function

2. Reactive force $P_{g}(t)$ of elastic supports

$$
P_{g}(t)=-\sum_{i=1}^{n g} C_{i} W\left(\ell_{i}, t\right) \delta\left(x-\ell_{i}\right)
$$

Total force system is

$$
P(x, z, t)=P_{\tau}(x, z, t)+P_{g}(t)
$$

The equation describing beam vibration including internal friction is

$$
E J\left(\frac{\partial^{4} W}{\partial x^{4}}+\alpha \frac{\partial^{5} W}{\partial x^{4} \partial t}\right)+\rho F\left(\frac{\partial^{2} W}{\partial t^{2}}+\beta \frac{\partial W}{\partial t}\right)=P(x, z, t)
$$

in which $\alpha$ and $\beta$ are constants characterizing for internal friction of beam. Substructure 1 bears the action of force $P_{d}^{\prime}(t)$ from the beam.

$$
P_{d}^{\prime}(t)=d \dot{W}_{\eta}(t)+k W_{\eta}(t)
$$

Here $W_{\eta}(t)$ and $\dot{W}_{\eta}(t)$ are deplacement and velocity of deplacement of beam at the contact position between body and beam. Besides it bears actions of gravity force $m g$ and force $G \sin \Omega t$. Thus total force system $P^{\prime}(t)$ acts on the body is

$$
P^{\prime}(t)=m g+G \sin \Omega t+d \dot{W}_{\eta}(t)+k W_{\eta}(t)
$$

The equation describing vibration of body has following form:

$$
m \ddot{z}+d \dot{z}+k z=m g+G \sin \Omega t+d \dot{W}_{\eta}(t)+k W_{\eta}(t)
$$

From that we have the equation system describing vibration of system

$$
\begin{aligned}
& G J\left(\frac{\partial^{4} W}{\partial x^{4}}+\alpha \frac{\partial^{5} W}{\partial x^{4} \partial t}\right)+\rho F\left(\frac{\partial^{2} W}{\partial t^{2}}+\beta \frac{\partial W}{\partial t}\right)=P(x, z, t) \\
& m \ddot{z}+d \dot{z}+k z=m g+G \sin \Omega t+d \dot{W}_{\eta}(t)+k W_{\eta}(t)
\end{aligned}
$$


with border condition

$$
\begin{array}{ll}
x=0 ; \quad W(0, t)=0 ; & \frac{\partial^{2} W(0, t)}{\partial x^{2}}=0 \\
x=\ell ; \quad W(\ell, t)=0 ; & \frac{\partial^{2} W(\ell, t)}{\partial x^{2}}=0
\end{array}
$$

This is equation system mixed of ordinary differential and particular differential. To solve it we transform it into ordinary differential equation system

\section{TRANSFORMATION INTO \\ ORDINARY DIFFERENTIAL EQUATION SYSTEM}

We will find solution of (2.8) under the form

$$
W(x, t)=\sum_{r=1}^{n} q_{r}(t) \sin (r \pi x / \ell)
$$

in which $q_{r}(t)$ are generalized coordinates we must determine. From (3.1) we have

$$
\begin{aligned}
& W_{\eta}(t)=W(\eta, t)= \sum_{r=1}^{n} q_{r}(t) \sin (r \pi \eta / \ell) \\
& \dot{W}_{\eta}(t)=\dot{W}(\eta, t)=\sum_{r=1}^{n} \dot{q}_{r}(t) \sin (r \pi \eta / \ell)+(\pi v / \ell) \sum_{r=1}^{n} r q_{r}(t) \cos (r \pi \eta / \ell) \\
& \frac{\partial W}{\partial t}=\sum_{r=1}^{n} \dot{q}_{r}(t) \sin (r \pi x / \ell) \\
& \frac{\partial^{2} W}{\partial t^{2}}=\sum_{r=1}^{n} \ddot{q}_{r}(t) \sin (r \pi x / \ell) \\
& \frac{\partial^{4} W}{\partial x^{4}}=\sum_{r=1}^{n}(r \pi / \ell)^{4} q_{r}(t) \sin (r \pi x / \ell) \\
& \frac{\partial^{5} W}{\partial x^{4} \partial t}=\sum_{r=1}^{n}(r \pi / \ell)^{4} \dot{q}_{r}(t) \sin (r \pi x / \ell)
\end{aligned}
$$

Replace (3.4) to (2.8) and set

$$
\begin{aligned}
& b_{r}=\left[E J \alpha(r \pi / \ell)^{4}+\rho F \beta\right] / \rho F \\
& e_{r}=E J(r \pi / \ell)^{4} / \rho F
\end{aligned}
$$

we have

$$
\sum_{r=1}^{n}\left\{\ddot{q}_{r}(t)+b_{r} \dot{q}_{r}(t)+e_{r} q_{r}(t)\right\} \sin (r \pi x / \ell)=(1 / \rho F) P(x, z, t)
$$

Integrate two member of (3.8) from 0 to $\ell$ after having multified them by $\sin (s \pi x / \ell)$ and noticing that

$$
\int_{0}^{\ell} \sin ^{2}(s \pi x / \ell) d x=\frac{1}{2} ; \quad \int_{0}^{\ell} \sin (r \pi x / \ell) \sin (s \pi x / \ell) d x=0 \quad \text { with } s \neq r
$$


we have

$$
\ddot{q}_{s}(t)+b_{s} \dot{q}_{s}(t)+e_{s} q_{s}(t)=\frac{2}{\ell \rho F} \int_{0}^{\ell} P(x, z, t) \sin (s \pi x / \ell) d x \quad(s=1, \ldots, n)
$$

Set

$$
\begin{aligned}
u_{r s} & =\frac{2}{\rho F \ell} \sum_{i=1}^{n g} C_{i} \sin \left(r \pi \ell_{i} / \ell\right) \sin \left(s \pi \ell_{i} / \ell\right) \\
f_{s}(t) & =\frac{2}{\rho F \ell}(m g+G \sin \Omega t) \sin (s \pi v t / \ell) \\
m_{s}(t) & =(2 m / \rho F \ell) \sin (s \pi v t / \ell) \\
a_{s}(t) & =(2 d / \rho F \ell) \sin (s \pi v t / \ell) \\
h_{s}(t) & =(2 k / \rho F \ell) \sin (s \pi v t / \ell)
\end{aligned}
$$

and integrate the right member of (3.9) equation we receive

$$
\frac{2}{\ell \rho F} \int_{0}^{1} P(x, z, t) \sin (s \pi x / \ell) d x=f_{s}(t)-m_{s}(t) \ddot{z}-a_{s}(t) \dot{z}-h_{s}(t) z-\sum_{r=1}^{n g} u_{r s} g_{r}(t)
$$

To replace this result in (3.9) and introduce symbols

$$
q_{n+1}=z ; \quad \dot{q}_{n+1}=\dot{z} ; \quad \ddot{q}_{n+1}=\ddot{z}
$$

we have

$$
\ddot{q}_{s}+m_{s}(t) \ddot{q}_{n+1}+b_{s} \dot{q}_{s}+a_{s}(t) \dot{q}_{n+1}+e_{s} q_{s}+\sum_{r=1}^{n g} u_{r s} q_{r}+h_{s}(t) q_{n+1}=f_{s}(t) \quad(s=1, \ldots, n)
$$

As to (2.9) equation, after replacing $\dot{W}_{\eta}, W_{\eta}$ by expression calculated from (3.3) and introduce following function

$$
\begin{aligned}
& \beta_{r}(t)=-(d / m) \sin (r \pi v t / \ell) \\
& \beta_{n+1}=d / m \\
& \gamma_{r}(t)=-[d r \pi v / m \ell) \cos (r \pi v t / \ell)+(k / m) \sin (r \pi v t / \ell) \\
& \gamma_{n+1}=k / m \\
& f_{n+1}(t)=g+(G / m) \sin (\Omega v t / \ell)
\end{aligned}
$$

we can write

$$
\ddot{q}_{n+1}=f_{n+1}(t)-\sum_{r=1}^{n} \beta_{r} \dot{q}_{r}-\sum_{r=1}^{n+1} \gamma_{r} q_{r}
$$

Replace (3.12) in (3.10), after some changes the equation system mixed of ordinary differential and particular differential $(2.8),(2.9)$ will be reduced into following ordinary differential equation system. 


$$
\begin{aligned}
\ddot{q}_{s}= & \sum_{r=1}^{n} m_{s} \beta_{r} \dot{q}_{r}-b_{s} \dot{q}_{s}+\left(m_{s} \beta_{n+1}-a_{s}\right) \dot{q}_{n+1} \\
& +\sum_{r=1}^{n}\left(m_{s} \gamma_{r}-u_{r s}\right) g_{r}-e_{s} q_{s}+\left(m_{s} \gamma_{n+1}-h_{s}\right) q_{n+1}+\left(f_{s}-m_{s} f_{n+1}\right) \quad(s=1, \ldots, n) \\
\ddot{q}_{n+1}= & -\sum_{r=1}^{n} \beta_{r} \dot{q}_{r}-\sum_{r=1}^{n+1} \gamma_{r} q_{r}+f_{n+1}(t)
\end{aligned}
$$

or we can write matrix form

$$
\ddot{\vec{q}}=\underline{B} \dot{\vec{q}}+\underline{C} \vec{q}+\dot{\vec{f}}
$$

in which $\underline{B}, \underline{C}$ are square matrix of $(n+1) \times(n+1)$ degree; $\vec{q}, \dot{\vec{q}}, \overrightarrow{\vec{q}}, \vec{f}$ are column vectors with $n+1$ elements

$$
\begin{aligned}
& \underline{B}=\left[\begin{array}{ccccc}
\left(m_{1} \beta_{1}-b_{1}\right) & m_{1} \beta_{2} & \cdots & m_{1} \beta_{n} & \left(m_{1} \beta_{n+1}-a_{1}\right) \\
m_{2} \beta_{2} & \left(m_{2} \beta_{2}-b_{2}\right) & \cdots & m_{2} \beta_{n} & \left(m_{2} \beta_{n+1}-a_{2}\right) \\
\vdots & \vdots & \ddots & \vdots & \vdots \\
m_{n} \beta_{1} & m_{n} \beta_{2} & \cdots & \left(m_{n} \beta_{n}-b_{n}\right) & \left(m_{n} \beta_{n+1}-a_{n}\right) \\
-\beta_{1} & -\beta_{2} & \cdots & -\beta_{n} & -\beta_{n+1}
\end{array}\right] \\
& \underline{C}=\left[\begin{array}{ccccc}
\left(m_{1} \gamma_{1}-u_{11}-e_{1}\right) & \left(m_{1} \gamma_{2}-u_{21}\right) & \ldots & \left(m_{1} \gamma_{n}-u_{n 1}\right) & \left(m_{1} \gamma_{n+1}-h_{1}\right) \\
\left(m_{2} \gamma_{1}-u_{12}\right) & \left(m_{2} \gamma_{2}-u_{22}-e_{2}\right) & \ldots & \left(m_{2} \gamma_{n}-u_{n 2}\right) & \left(m_{2} \gamma_{n+1}-h_{2}\right) \\
\vdots & \vdots & \ddots & \vdots & \vdots \\
\left(m_{n} \gamma_{1}-u_{1 n}\right) & \left(m_{n} \gamma_{2}-u_{2 n}\right) & \ldots & \left(m_{n} \gamma_{n}-u_{n n}-e_{n}\right) & \left(m_{1} \gamma_{n+1}-h_{n}\right) \\
-\gamma_{1} & -\gamma_{2} & \ldots & -\gamma_{n} & -\gamma_{n+1}
\end{array}\right] \\
& \vec{f}=\left[\begin{array}{c}
f_{1}-m_{1} f_{n+1} \\
f_{2}-m_{2} f_{n+1} \\
\vdots \\
f_{n}-m_{n} f_{n+1} \\
f_{n+1}
\end{array}\right], \quad \vec{q}=\left[\begin{array}{c}
q_{1} \\
q_{2} \\
\vdots \\
q_{n} \\
q_{n+1}
\end{array}\right], \quad \dot{\vec{q}}=\left[\begin{array}{c}
\dot{q}_{1} \\
\dot{q}_{2} \\
\vdots \\
\dot{q}_{n} \\
\dot{q}_{n+1}
\end{array}\right], \quad \ddot{\vec{q}}=\left[\begin{array}{c}
\ddot{q}_{1} \\
\ddot{q}_{2} \\
\vdots \\
\ddot{q}_{n} \\
\ddot{q}_{n+1}
\end{array}\right]
\end{aligned}
$$

Solving the (3.14) we find out generalized coordinates $q_{s}$ then replacing in (3.1) we will determine the dynamic flexure $W(x, t)$

Dynamic stress is calculated by the formula

$$
\sigma(x, t)=-\frac{E J}{M_{k u}} \frac{\partial^{2} W(x, t)}{\partial x^{2}}=\frac{E J r^{2} \pi^{2}}{\ell^{2} M_{k u}} \sum_{r=1}^{n} q_{r}(t) \sin (r \pi x / \ell)
$$

In which $M_{k u}$ is against-flexing moment of beam

\section{BEAM PROGRAM AND CALCULATING EXAMPLE}

Based on this algorithm, we have built calculating program BEAM by Turbo Pascal 6.0 language. The program can be operated on every PC suitable to IBM PC. Results are both showed on drawing display and printed clearly in the printer. Communication with the user by menu is very flexible. 
Using this program, we have calculated many series of examples which give rather exact results. Here under is an example.

Calculating model is drawn on figure 4.1 with following data $\ell=32000.00 \mathrm{~mm} ; \rho F=10 \mathrm{~kg} / \mathrm{mm}$; $M_{k u}=1400000000000$ n.mm.mm; $\alpha=0 ; \beta=0.001 ; n g=3 ; \ell_{1}=8000 \mathrm{~mm} ; C_{1}=17160000$ $\mathrm{N} / \mathrm{mm} ; \ell_{2}=16000 \mathrm{~mm} ; C_{2}=17000000 \mathrm{~N} / \mathrm{mm} ; \ell_{3}=24000 \mathrm{~mm} ; C_{3}=17000000 \mathrm{~N} / \mathrm{mm} ;$ $v=10000 \mathrm{~mm} / \mathrm{s} ; m=10000 \mathrm{~kg} ; d=110 \mathrm{~N} . \mathrm{s} / \mathrm{mm} ; g=9800 \mathrm{~mm} / \mathrm{s}^{2} ; \Omega=2.5 \mathrm{Rad} / \mathrm{s} ; G=5 \mathrm{~mm} ;$ $n=4$.

Time in which the body passes through beam is devided into 100 points of time and studying 32 sections of beam.

\begin{tabular}{lllllll}
\hline & Time point 15 & Time point 38 & Time point 63 & Time point 90 \\
\cline { 1 - 1 } & $0.20297090 \mathrm{~mm}$ & $1.55595175 \mathrm{~mm}$ & $1.46113285 \mathrm{~mm}$ & $3.13275450 \mathrm{~mm}$ \\
\hline
\end{tabular}

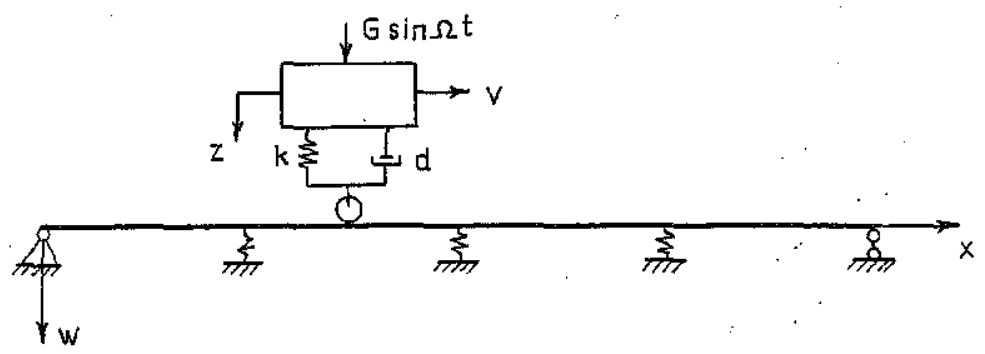

Fig. 4.1

Figure 4.2 shows the dynamic flexure of beam at 4 times points 15, 38, 63 and 90 .

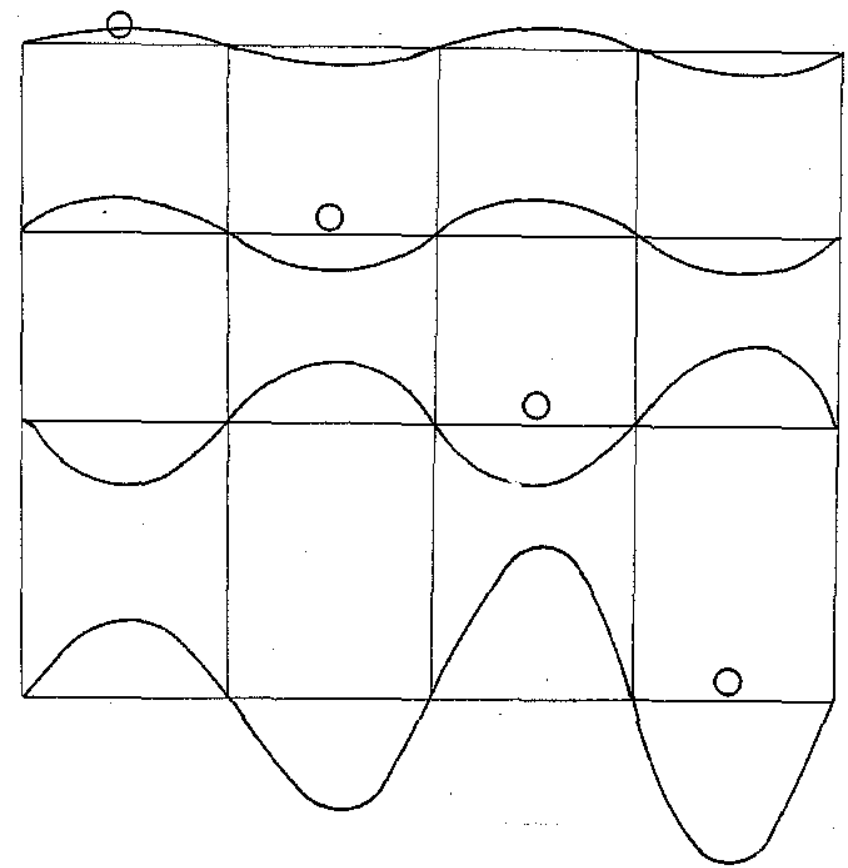

Fig. 4.2 


\section{CONCLUSION}

Applying the method of separating structure has established the equation system of bending of continuous beam with elastic intermediate supports under the action of moving body and set up at the same time the algorithm and one computing program (BEAM). The BEAM program can be used for calculating bridges bearing moving load.

This publication is completed with financial support from the National Basic Research Program Natural Sciences.

\section{REFERENCES}

1. Đỗ Xuân Thọ; Nguyễn Văn Khang. Tính toán bằng số dao động uốn cưa dầm liên tục dưới tác dụng cưa tải trọng di động. Tạp chí Cơ học, số 3, 1992.

2. Gasch R., Knothe K. Strukturdynamik, Band I und Band II, Berlin, Heidelberg, New York, Springer - Verlag 1987.

3. Филиппов А. П., Кохманюк С. С. Динамическое воздейтвие подвижных нагрузок на цтержни. Изд. Наукова Думка, Киев, 1967.

4. Филиппов А. П., Кохманюк С. С., Воробыв Ю. С. Воздействие динамических нагрузок на әлементы конструкцин. Изд. Наукова думка, Киев, 1974.

Received March 21, 1995

\section{DAO ĐộNG UỐN THAM SÓ CỬA DẦM LIÊN TỰC TRÊN GỐI ĐAN HỒI TRUNG GIAN DƯỚI TÁC DỤNG CƯA VẬT THỂ DI ĐộNG}

Trong bài này áp dụng phương pháp các cấu trúc con (substructurs) [2] đã thiết lập phương trình dao động cừ dầm dưới tác dụng của vật thể di động. Tiếp theo đã xây dựng thuật toán để giai hệ phương trình dao động nhận được. Từ thuật toán này một chương trình máy tính đã được lập bằng ngôn ngữ TURBO PASCAL 6.0. 